\title{
Mindreading, Communication and the Learning of Names for Things
}

\author{
PAUL BLOOM
}

\begin{abstract}
Children are strikingly good at learning the meanings of names for things. There is considerable evidence that they solve this mapping problem through inferring the referential intentions of other people. This mindreading capacity might also underlie children's assumptions that words do not overlap in reference, and that new words are likely to refer to whole objects. Finally, there is some support for the view that the mindreading that underlies word learning is due to a general capacity to infer mental states, not a submodule that is dedicated to communication.
\end{abstract}

There are two facts about word learning that everyone accepts. The first is that words really do have to be learned. There is controversy over how much conceptual structure and linguistic knowledge is innate, but nobody thinks that this is the case for the specific mappings between sounds (or signs) and meanings. This is because these mappings vary arbitrarily from culture to culture. No matter how intelligent a British baby is, for instance, she still has to learn, by attending to the language of the people around her, that rabbits are called 'rabbits', that sleeping is called 'sleeping', and so on.

The second fact is that children are strikingly good at this sort of learning. Even by conservative estimates, English-speaking adults know in the neighborhood of 60,000 words. Since children start learning words at about their first birthday, this means they have to learn, on average, ten new words a day. Words are learned by abused and neglected children (children who are not taught words and not encouraged to learn them) and they are learned as well by children raised in societies in which there is considerably less direct interaction between children and their parents (see Bloom, 2000 for review).

Children's skill at word learning has been explored experimentally. There are several studies in which children are exposed to new words under a variety of different circumstances and later tested on their memory of the words' meanings. In one such study, Lori Markson and I (Markson and Bloom, 1997) exposed 3-year-olds and 4-year-olds to a new word by playing a game with them in which some objects were used to measure other objects. There were

Thanks to Dare Baldwin, Ruth Campbell, and Deirdre Wilson for helpful comments on an earlier version of this paper.

Address for correspondence: Department of Psychology, Yale University, P.O. Box 208205 New Haven, CT 06520-8205, USA.

Email: paul.bloom@yale.edu

Mind \& Language, Vol. 17 Nos 1 and 2 February/April 2002, pp. 37-54.

(c) Blackwell Publishers Ltd. 2002, 108 Cowley Road, Oxford, OX4 1JF, UK and 350 Main Street, Malden, MA 02148, USA. 
ten objects in this game, and the children played equally long with each of the objects. The word was introduced by saying, for one of the objects: 'Let's use the koba to measure which is longer . . We can put the koba away now'. Children were not asked to repeat the word, and were not tested to see if they were even paying attention to what the adult was saying.

We tested the children a month later, by bringing out the same ten objects and asking them to 'Show me the koba'. If children forgot the word, or had never attended to it in the first place, they should get this right about $10 \%$ of the time (by guessing). In fact, even 3-year-olds point to the right object over half of the time. There were no age differences-3-year-olds did just as well as 4-year-olds, and just as well as adults. This ability to successfully learn words under conditions of minimal exposure has been dubbed 'fast mapping' (Carey and Bartlett, 1978), and this capacity has been found in 2-year-olds (e.g. Markson, 1999) and even in 1-year-olds (e.g. Woodward et al., 1994).

These two facts about word learning - that words are learned, and that children are good at learning them-are uncontroversial. But little else is. The rest of this paper concerns an issue of particularly heated debate. In order to learn language, children must be capable of learning the names of familiar middle-sized objects. They must be able to figure out, in the study described above, which thing is the koba, and, in the real world, which objects are rabbits, which person is Mom, and so on. This is only part of the problem of word learning, of course. Children also have to learn words that are not object names, such as determiners, prepositions, verbs, and abstract nouns. But this learning of simple object names is fundamental. If children are to start learning words - and, indeed, to start learning language - they need to begin by learning names for things.

In what follows, I will argue that even young children solve this problem through inferring the referential intentions of other people. Hence word learning involves what has variously been called naïve psychology, theory of mind, and mindreading. I will then make some speculations about the origin and nature of these mindreading capacities, and how they relate to other, nonlinguistic capacities.

\section{Solving the Mapping Problem}

How do children figure out that rabbits are called 'rabbits'? The traditional answer to this question, in both psychology and philosophy, is that they do so because they possess general associative learning mechanisms. These guide children to establish the mapping between words and what they refer to. This works because the environment is amenable to a statistical learning process; because words are typically spoken at the same time that the child is focused on what the words refer to. And this is because adults tailor their speech to the attentional focus of their children. As John Locke (1690, p. 108) put it: 'To make children understand what the names of simple ideas or substances 
stand for; people ordinarily show them the thing whereof they would have them have the idea and then repeat to them the name that stands for it.' For example, a child will be regarding a rabbit, and as she does so, she will hear an adult say 'rabbit'. Through general principles of associative learning, the sound and the image become associated in the child's mind, so that one evokes the other, and the child could be said to have learned the meaning of the word.

Locke's view is still dominant in contemporary thought. For instance, Lois Bloom (1994, p. 221) states that 'Word learning consists of good old-fashioned associative learning ... The meanings of early words like cookie, gone, more, and mama, or little sentences like "eat meat" or "throw ball" can be gotten from the words and their corresponding events'. And Kim Plunkett (1997, p. 146) suggests 'Word learning ... can be driven by the interaction of general learning mechanisms, highly sensitive to particular statistical regularities in the input, with a richly structured environment'.

Who could doubt this? It seems perfectly reasonable to assume that words are learned because the child attends to what they co-occur with. This theory also has the advantage of explaining the process of word learning in terms of learning mechanisms that plainly exist (nobody doubts that humans can learn co-occurrence relationships), that have been studied for hundreds of years, and that we share with virtually all other species.

But there are other possibilities. The most promising alternative was presented by Saint Augustine, about a thousand years before Locke. In his Confessions, he considers a similar situation to that of Locke, one in which a child observes an act of ostensive naming by an adult. But Augustine tells a very different story of how the word is learned. Instead of passively associating sound and image, the child actively tries to figure out the meaning that the adult had intended to express. And this is done through attending to cues to the intention of the speaker: "When [my elders] named any thing. . I saw and remembered that they called what they would point out by the name they uttered. And that they meant this thing and no other was plain from the motion of their body. . indicating the affections of the mind, as it pursues, possesses, rejects, or shuns.' (398, p. 11).

Both Locke and Augustine agree that the ideal circumstance for learning a new object name is to regard the object at the moment that you hear the name. Nobody doubts that if you want to teach a child the word rabbit, the very best way to do so is to wait until there is a rabbit around and then point and present the name for it: 'Hey, look at the rabbit. That's a rabbit!' But why is this situation so ideal? Locke believes the child is passively connecting the word and the percept, and so ostensive naming provides the best correspondence between word and percept - the situation is the richest from a statistical perspective; Augustine sees the child as trying to make sense of what the adult is intending to refer to, and from this perspective, ostensive naming provides the best source of intentional information - if an adult is pointing to an object 
and saying its name, it is an excellent bet that the adult intends the word to refer to that object.

There are several considerations that favor Augustine's theory of mind perspective over Locke's associationist perspective. These are presented in detail in Bloom (2000), and I will summarize three of the main arguments below.

The first has to do with the input that children receive. Children do not need ostensive naming for word learning. We know this because such a pattern of naming is not a human universal. For example, Schieffelin (1985) describes the cultural context of children acquiring Kaluli. These children grow up in a rich linguistic environment, surrounded by adults and older children who are talking to one another, including making observations about the infant himself-but there is no naming of objects and no labeling interactions. And when a child names an object for an adult, the adult's response is disinterest. This lack of object labeling has been observed in other cultures as well (Lieven, 1994). But all normal children learn words, regardless of the culture in which they are raised.

Even for Locke's own culture, the relationship between the word and the world is nowhere near as transparent as he thought it was (see Gleitman, 1990). It is true that Western parents often use words to refer to what the child is attending to at the moment the word is spoken. But they often fail to do so. Some of the time children hear 'Look at the rabbit!' for instance, they will be looking at a rabbit. But some of the time they hear 'Look at the rabbit!' they will be looking at a shoe or at their mother's face. If Locke were right, one would expect serious mistakes — such as a child thinking that rabbit means face-to occur at least some of the time. But such mistakes never occur.

To account for this error-free learning, an associationist might argue that children are inherently cautious, and only use a word when they have adequate statistical evidence for its meaning, such as hearing the word with a consistent referent a dozen times, across suitably different situations. The logic here is reasonable, but the premise is empirically false: Children do not wait-they can learn a word after hearing it used a few times in a single situation, as in the fast mapping study described above. The fact that object name acquisition is both fast and errorless suggests that it is not the product of an associationist process.

The second consideration in favor of the theory of mind view comes from experimental research. In a fascinating series of studies, Dare Baldwin (1991, 1993) tested children in a context in which they were given one object to play with while another object was put into a bucket that was in front of the experimenter. When the child was looking at the object in front of her, the experimenter looked at the object in the bucket and said a new word, such as 'It's a modi!' This gives rise to a perfect Lockean correspondence between the new word and the object the child was looking at. But 18-month-olds do not take modi as naming this object. Instead they look at the experimenter and redirect their attention to what she is looking at, in this case, at the object 
in the bucket. And when later shown the two objects and asked to 'find the modi', a child will assume that the word refers to the object the experimenter was looking at when she said the word-not the object that the child herself was looking at.

These studies show that contiguity between word and percept is not necessary for word learning. Further work suggests that contiguity is also not sufficient. In another study, 15-20-month-olds were alone in a room with a novel object. When they looked at the object, they heard a disembodied voice (from a hidden adult outside the room) saying something such as 'Dawnoo! There's a dawnoo!' Under these circumstances, they did not learn the word (Baldwin et al., 1996). That is, even with a perfect association between hearing a word and attending to an object, young children will make the connection only if they believe that it is an act of naming-and for this, the speaker has to be present. (Adults, of course, could learn the word in the above situation, not because we are more associationist than children, but because we would infer that the disembodied voice is actually an act of naming by a person we cannot see.)

As a further example, Susan Birch and I (Birch and Bloom, in press) explored children's ability to assess the knowledge of other people when inferring reference. The children were shown two bags of animals, and were told that one bag contains animals that were familiar to the experimenter, and that the other bag contains unfamiliar animals, those that the experimenter had never seen before. Then the experimenter took one animal from each bag (such as one rabbit from each bag), put a box in front of her, covered her eyes, and asked the child to: 'Put Jesse in the box'. The adult intuition here is that 'Jesse' is more likely to be the familiar rabbit, the one that the experimenter knows. This is because knowing the proper name of an individual requires some sort of acquaintance with that individual. As predicted, even 2year-olds assume that the proper name refers to the familiar item, providing further evidence for the role of theory of mind in early word learning.

The final consideration in favor of the theory of mind perspective comes from the study of language development in autistic children. Autism is a developmental disorder that is characterized by a range of deficits, including impairments of socialization, communication, and imagination. One prominent theory of autism is that this cluster of deficits is the product of a delayed, impaired or non-existent theory of mind (Baron-Cohen, Leslie and Frith, 1985). This elegantly explains the range of specific problems that autistic children have, including difficulties with understanding false belief, deception, and ignorance, while at the same time accounting for preserved abilities in other domains.

If theory of mind is central to solving the mapping problem, it should follow that autistic individuals, who are impaired in theory of mind, should show a corresponding impairment in word learning. And they do. Indeed, about $30 \%$ of the individuals who are labeled as autistic have no language at 
all. They might first appear to be deaf, since they often fail to orient to speech, and they sometimes produce odd vocalizations that do not resemble speech or babbling.

Other autistic individuals have some language, but it is unusual. Children with autism will often show pronominal reversal, using 'I' for 'you' and viceversa, and might use entire phrases in a parroted way; for instance, saying, 'Do you want a biscuit?' to mean 'I want a biscuit.' Words and phrases are used in a 'simple associative way' (Frith and Happé, 1994), so that 'Apple' might always mean, 'Give me an apple.' There is also anecdotal evidence of unusual errors. Kanner (1943) reported an autistic boy who used the phrase 'Peter eater' to talk about saucepans. His mother explained this by recounting that when he was two years old, she was reciting the rhyme 'Peter, Peter, Pumpkin Eater' to him when she dropped a saucepan with a loud clatter. Baron-Cohen, Baldwin and Crowson (1997) discuss an autistic toddler who would call a toy truck 'a sausage', apparently because his mother had said 'Tommy, come and eat your sausage' as the boy was looking at his truck. Some of this odd linguistic behavior can be readily explained as the perils of adopting an associationist approach to language learning.

To explore this issue experimentally, Baron-Cohen et al. (1997) studied autistic children using the discrepant looking paradigm of Baldwin (1991) discussed above, in which an experimenter looks at one object and utters its name while a child is attending to another object. A purely associative mechanism would lead children to map the word onto what they are attending to, while learning based on theory of mind would lead them to map the word onto what the experimenter is looking at. As predicted, autistic children assumed that the word referred to the object they were attending to, while both normal children and mentally handicapped children, matched to the autistic group in mental age, assumed that the word referred to the object that the speaker was attending to.

One can make the autism argument in two ways, as both a positive argument in favor of the theory of mind approach and as a negative argument against the associationist approach. The positive argument is that if theory of mind is central to word learning, it should follow that those same individuals who have a general deficit in theory of mind should be poor at word learning. This prediction seems to be supported (though see below for discussion of some potential exceptions). The negative argument is that an associationist theory predicts that any sufficiently powerful general-learning machine should be able to learn words. This is falsified in the case of some individuals with autism - they are very smart and very capable of learning in non-communicative domains. But they do not learn the meanings of word in the same way that children do. 


\section{Expanding the Role of Mindreading}

How far can one take a theory of mind account? How much of word learning can be explained by mindreading? Elsewhere I have argued that children's naming of visual representations (Bloom, 2000; Bloom and Markson, 1998) and artifacts more generally (Bloom, 1996, 2000; Gelman and Bloom, 2000) is rooted in intentional attribution. Here I will suggest that two basic facts about how children learn object names can also be explained in terms of theory of mind.

The bias against lexical overlap Imagine you are shown two objects, a familiar one, such as a rabbit, and a novel one, such as a strange machine, and you are asked to 'Point to the fendle'. Which object do you point to?

Most likely, you would not point to the rabbit; you would instead point to the novel object. Markman and Wachtel (1998) find that young children do the same, and they explain this in terms of a 'mutual exclusivity' principle that biases them to think that words should not have overlapping reference. If an object already has a name-such as rabbit - then another word is likely not to name that object, but instead to name another object. (If there is no other object present, we tend to think the word refers to a part of the object, or to one of its properties.)

Where does this bias come from? It might be a specifically linguistic phenomenon, a fact about words that is either innate or acquired in the course of language development (e.g., Mervis, Golinkoff and Bertrand, 1994). Or it could be the product of a general principle of learning, one guiding children to prefer one-to-one mappings as part of a general tendency to exaggerate regularities. (e.g., Markman, 1992). (Note in either case, it could not be an absolute constraint, since natural languages in fact do give the same object many names - a single object can be 'Flopsy', 'a rabbit', 'an animal', 'a pet', and so on.)

A different possibility, which I will explore here, is that mutual exclusivity is a product of theory of mind (e.g., Clark, 1997). The proposal here is that listeners expect speakers to be informative, and so they expect speakers to use familiar words to refer to familiar things. Going back to the example above, you may have reasoned as follows (implicitly, of course).

'It is clear that he means me to point to one of the two objects. If he meant me to point to the rabbit, he would have referred to the rabbit in some way that would have made sense to me-he would have asked for "the rabbit", for instance. But he didn't; he used a novel word. And so he doesn't want me to point to the rabbit. The only remaining option is that strange object, so this must be what 'fendle' refers to.' 
How can we test this? If it is true that children's bias against lexical overlap has to do with intuitions about speaker's intent, then this bias should not be limited to words. It should extend to any sort of communicative information, any discourse event. After all, the claim here is that the refusal to map 'fendle' onto the rabbit is not special to the lexicon, nor is it general enough to apply to all mappings. Rather it involves expectations about communication, a general belief that if someone wants to tell you about something, he or she will do so in a way that you will understand. To put it differently, it falls out from a general expectation that utterances will be 'relevant' (see Sperber and Wilson, 1986).

This prediction was tested by Diesendruck and Markson (2001). In one part of the study, three-year-olds were presented with two unfamiliar objects and given a novel name for one of them ('This is a mep'). Then they were asked: 'Can you show me a jop?'. They tended to think that 'jop' referred to the unnamed object, replicating previous research. The other condition is the interesting one. Here a different group of children were shown the very same two objects, told a novel fact about one of them ('My sister gave this to me.') and were then asked to select the referent of a different fact ("Can you show me the one that dogs like to play with?'). Once again, children tended to choose the other object as the referent of the new information. This is presumably because they were reasoning as follows:

'It is clear that he means me to point to one of the two objects. If he meant me to point to the object that his sister gave him, he would have referred to it in some way that would make sense to me-he would have asked for "the one my sister gave me", for instance. But he didn't; he used a novel fact. And so he doesn't want me to point to the first object. The only remaining option is that second object, so this must be what the novel fact refers to.'

Diesendruck and Markson went on to test a further prediction: If children are using pragmatic reasoning about the adult's intentions in using the new fact, they should be less inclined to produce such a response in a two-speaker scenario, where the second speaker lacks mutual knowledge with the child. That is, if one speaker tells the child 'My sister gave this to me' about one object, and then a different speaker, new to the discourse context, enters the room and asks 'Can you give me the one that dogs like to play with?', the prediction is that children should now choose each of the objects with equal frequency. This is precisely what occurred.

Taken together, these findings support the notion that lexical contrast has its origin in children's expectations about the communicative behavior of others. It applies just as strongly when children are taught facts about objects as when they are taught words, and it does not apply when the pragmatic expectations are modified, as in the two-speaker condition. The results from (C) Blackwell Publishers Ltd. 2002 
the two-speaker condition also show that it is not the result of a general preference for one-to-one mappings.

This is not to deny that in certain regards words really are different from facts. For one thing, words have public meanings. If one person says of an object 'My sister gave this to me', there is no reason to expect this fact to affect the linguistic behavior of someone who later arrives on the scene. But if one person describes an object as a 'mep', it would be reasonable for a child to infer that other people know this word as well. Hence when a second person, new to the discourse content, asks for the 'jop', children could infer that if she meant to refer to the mep, she would have asked for it. Since she did not, she must have meant to refer to the second object. (This analysis raises the question of how children come to understand that words have this special property of having public meanings, something discussed in Bloom 2000.)

The whole object bias Once a child hears a new word and knows that it is used to refer to a given rabbit, how does he or she determine the precise meaning of the word? From a logical standpoint, there is an infinite number of possible meanings the word might have (Goodman, 1983; Quine, 1960). Even restricting ourselves to those meanings that are conceptually accessible to the child, and which correspond to words in natural languages, there are many plausible interpretations. The word could refer to the whole object (as in 'rabbit'), a part of the rabbit ('tail'), a property of the rabbit ('white'), the action that the rabbit is performing ('running'), and so on. How does the child figure out which interpretation is the right one?

There are several cues children could use, including the syntax of the word and the sorts of entities that are present in the immediate environment, but it is clear that children have a natural default hypothesis. In the absence of other information, they take the word as referring to the whole object. Object names constitute a larger proportion of children's early vocabularies than they do of the vocabularies of older children and adults, something which is true in every language and culture that has been studied (Gentner and Boroditsky, 2001). And when you show children an object and give them a name for it, they tend to assume that the name refers to the whole object, not to a part, a property, or anything else (e.g., Markman, 1989). As John Macnamara (1972, p. 11) put it, 'It is obvious that an infant has the capacity to distinguish from the rest of the physical environment an object which his mother draws to his attention and names. It seems clear too that in such circumstances he adopts the strategy of taking the word he hears as a name for the object as a whole rather than as a subset of its properties, or for its position, or weight, or worth, or anything else.'

There are several theories as to why this bias exists, but I want to discuss just two of them here. First, the whole object bias could be conceptual. We naturally see the world as containing objects and so, when we hear new words and have to figure out what they refer to, objects are natural candidates. In 


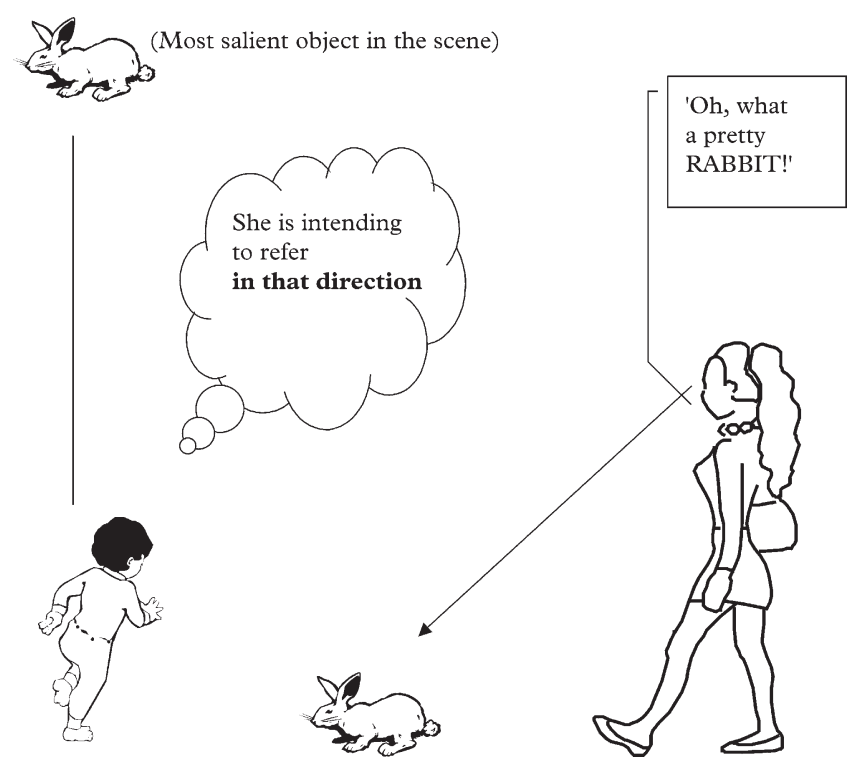

Figure 1

other words, we are predisposed to view words as describing whole objects because we are predisposed to think about the world as containing whole objects. Even babies parse the word into distinct objects, and can track and enumerate objects (Spelke, 1994). Once children know that words are used to refer, objects are the natural candidates for what they are referring to (see also Gentner, 1982; Macnamara, 1982; Maratsos, 1991).

A second proposal is that the whole object bias could be based on theory of mind: Children and adults might believe that other people, when they use words, typically intend to refer to objects (e.g., Baldwin, 1995; Tomasello and Akhtar, 1995). The distinction between the two proposals is illustrated in Figure 1 (the conceptual bias proposal) and Figure 2 (the intentional bias proposal). As a third option, it might be that children attend to objects when learning new words because objects are salient individuals and that children attend to objects when learning new words because they believe that adults intend to refer to objects. This hybrid proposal is shown in Figure 3.

The reason to take this third option seriously is that the conceptual bias and the intentional bias could be related. The bias in intentional interpretation may be a consequence of the conceptual bias. In particular, the reason that children believe adults are prone to attend to objects might be because children themselves are prone to attend to objects. This is not to say that children have an explicit belief that others will share their focus of attention. It is just that objects are plainly salient to children-and so, when inferring the referential intentions of other people, they might naturally (and correctly) assume that (C) Blackwell Publishers Ltd. 2002 


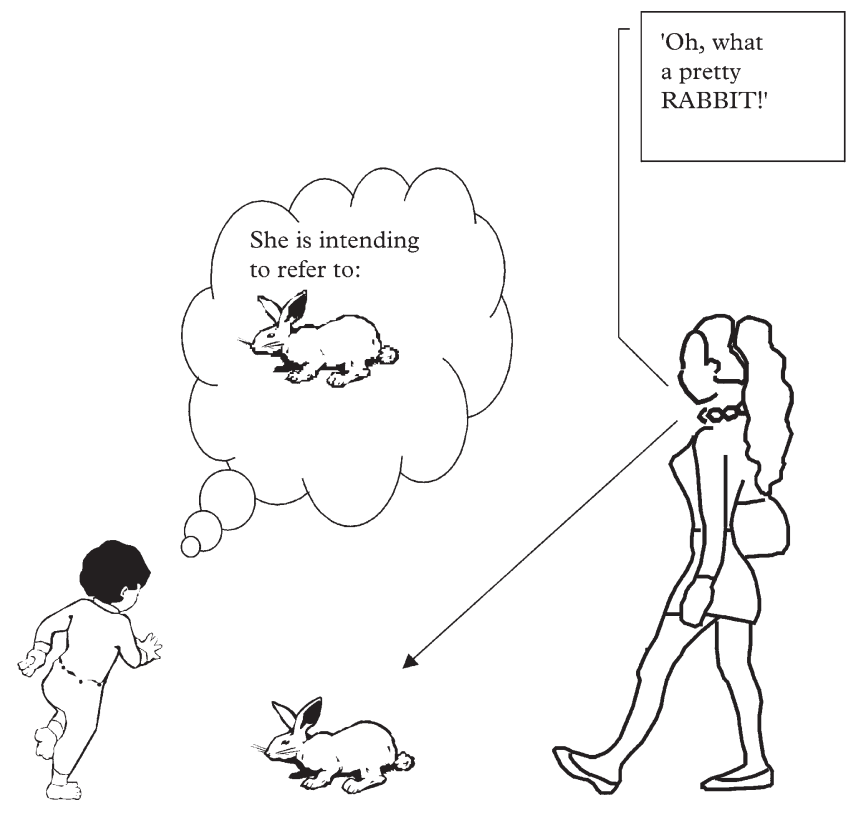

Figure 2

they are salient to others as well. As a result, they will infer that others, when using new words, are likely to refer to whole objects. After all, there is an abundant literature within social psychology showing that we tacitly assume that others share our beliefs, knowledge, and so on-even when this assumption is not warranted. This tendency, if anything, is exaggerated in children, due to their processing limitations.

There is considerable support for the conceptual proposal; even outside the context of language, babies and children find objects to be highly salient (see Bloom, 2000). What about the intentional proposal? This is less clear. It is not difficult to modify the context so as to override the whole object bias. For instance, one can use pointing to motivate 2-year-olds to treat a novel name as referring not to a whole unfamiliar object, but to a part of the unfamiliar object (Kobayashi, 1998). Or one can manipulate piles of objects so that a new word is interpreted not as an object label, but as a collective noun, a word like 'forest' or 'flock' (Bloom, 1994). Perhaps these manipulations work because they shift the child's interpretation of the intention of the speaker. On the other hand, they might work by simply shifting the focus of the child's own attention. Until the appropriate experimental tests can be devised, it is an open question which of the three theories of the whole object bias-conceptual, intentional or hybrid-is the correct one. 


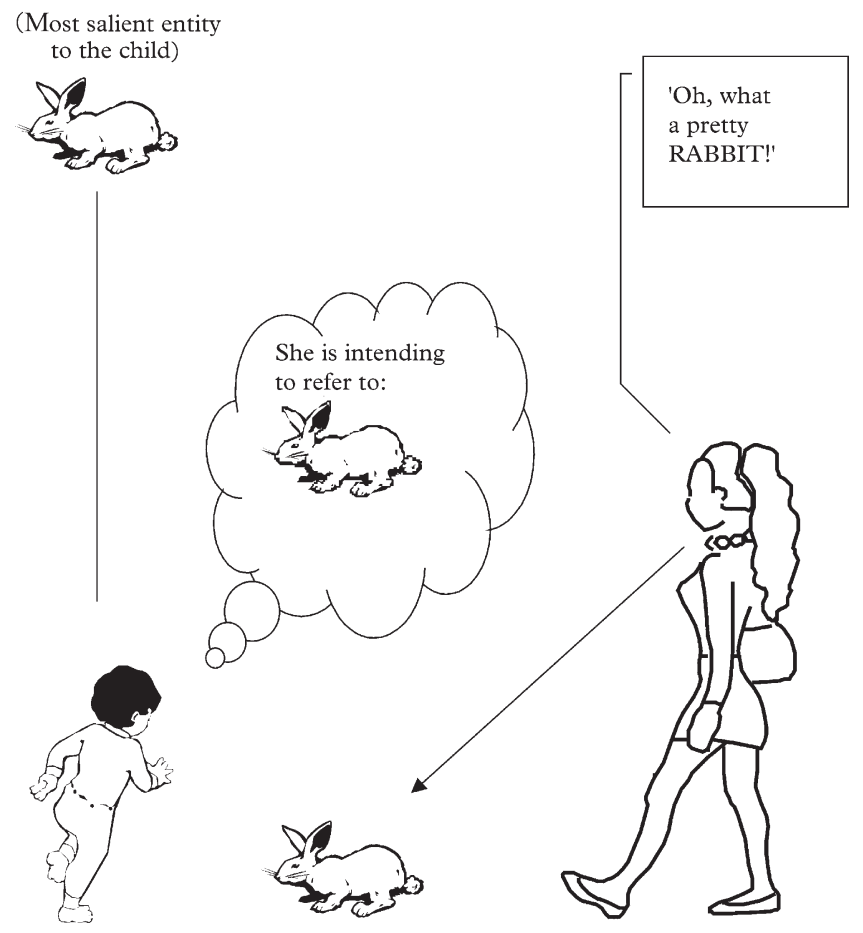

Figure 3

\section{Where Do These Mindreading Abilities Come From?}

If one agrees that theory of mind is essential to word learning, this raises the further question of the nature of this capacity. One can distinguish two alternatives. One is that there is a single capacity for mindreading that applies across several domains, including communication. This is the position taken by many researchers who study pragmatics - the inferences made in the course of producing and understanding language are nothing more than a more general mindreading ability applied to a specific domain (e.g., Grice, 1975). If we extend this account to apply to the learning of words, it would imply that the capacity for mindreading that underlies children's learning of object names is the very same one that underlies our understanding of the beliefs and desires of other people more generally. Hence the use of mindreading to learn words is not an adaptation. It is, in Darwin's term, a 'preadaptation'. We are using something that has evolved for one purpose (to figure out the thoughts of other people) for another purpose (word learning).

An alternative, proposed by Sperber (2000) and Sperber and Wilson (this volume), is that the mindreading ability that underlies language ability does not arise from a more general capacity to reason about mental states. It is instead the product of an evolved sub-module of the mindreading faculty, one

(C) Blackwell Publishers Ltd. 2002 


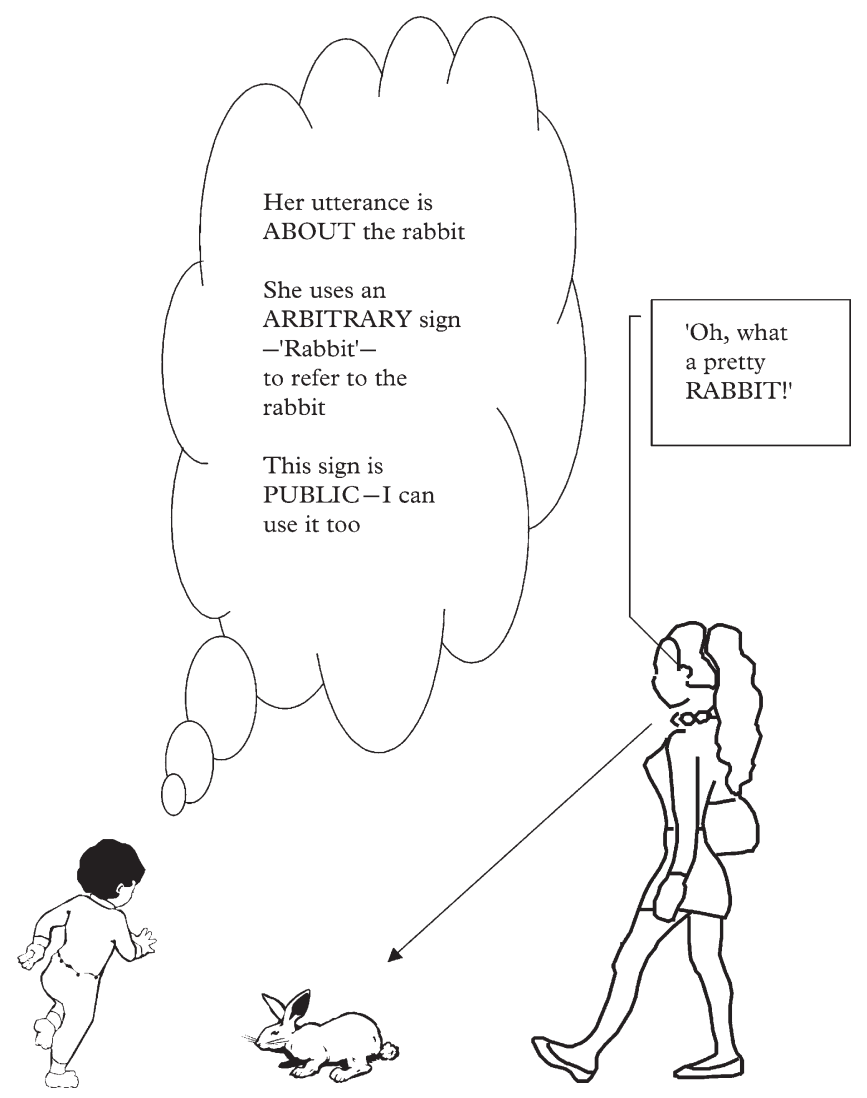

Figure 4

specially adapted to the problem of communication, including, perhaps, the problem of word learning. Not a theory of mind in general, but a 'theory of communication'.

One way to explore these issues is to ask whether the same sorts of intentional attribution that go into making sense of a new word apply in other non-communicative domains. Consider the analysis shown in Figure 4.

To learn the word rabbit, the child must tacitly know, at minimum, the following: The word is used to refer to the rabbit (it is about the rabbit), its connection to the rabbit is arbitrary (there is nothing rabbit-like about the word 'rabbit') and it is public (anyone, including the child, can use rabbit to refer to rabbits.)

Interestingly, names are not the only things that have these properties. Consider first reference. One can refer to something with a pronoun ('Look at that'), a gesture, or a point. Non-communicative mental states can also be about things in the world. If I look at an object on the ground, and my nose 
scrunches up in disgust, it is reasonable to infer that my disgust is about the object on the ground. And young children can make such an inference. They can infer, for instance, that an adult's emotional response-such as disgustis about the object that the adult is attending to at the time she makes the response (Baldwin and Moses, 1994). More generally, children quickly develop a tacit understanding that adults are usually thinking about what they are looking at.

What about the arbitrary and public nature of language? A sensitivity to both of these properties is revealed in the imitation research of Meltzoff, (1988, 1995). In one study, 14-month-olds are shown an unusual-and arbitraryact that achieves a goal, as when an adult bends at the waist to touch a panel with her forehead, causing a light to go on. When shown this, babies will often spontaneously imitate the act, appreciating its public nature. In other words, they can observe a novel and arbitrary act and then generate that act itself. It does not seem like too much of a stretch to claim that this same capacity underlies children's ability to hear a novel word used to refer to an object, and then use the word themselves, for the same purpose.

In sum, the same inferential capacities relevant to communication apply as well to non-communicative situations. The same direction of gaze cues that children use when figuring out what object someone is labeling (Baldwin, 1991) are used to figure out which object someone is disgusted by (Baldwin and Moses, 1994). The same capacity required to appreciate the arbitrary and public nature of language (Saussure, 1916) might well underlie the understanding of goal-directed action in general (Meltzoff, 1988, 1995). It is highly suggestive as well that these non-communicative aspects of theory of mind emerge in normal children at roughly the same age at which children begin to learn words. All of this is consistent with the theory that the mindreading ability used in language is the same as used in intentional attribution more generally, and is not the product of a distinct module or submodule.

This is not to deny, of course, that children grow to become especially fluent at intentional attribution in the domain of language, perhaps more so than in any other domain. But this is not strong evidence for a distinct module, since it is just as compatible with a practice effect. After all, children do not start off learning ten words a day; they begin learning words at a much slower rate, and gradually speed up over the course of development (see Bloom, 2000). The same might hold for pragmatics more generally. Children might start slow, inferring speaker's intent only through an effortful process, but, over the course of years of practice, across diverse situations, they become much better at it, increasingly able to perform complex attributions accurately, quickly, and unconsciously.

As an analogy, A. Alvarez (2001, p. 67) discusses the game of poker, pointing out that 'one of the many gifts that separates the professionals from the amateurs is the ability to read their opponents' hands with uncanny accuracy from tiniest clues: timing, position, the way their fingers move their chips or

(C) Blackwell Publishers Ltd. 2002 
their eyes flicker, even the pulse beat in their neck'. As he puts it, a professional poker player doesn't play his cards, he plays the other players. This gift is special to poker; people who make their living at the game are no better than the rest of us at mindreading in everyday life. But surely there is not a submodule of the mindreading capacity dedicated to poker; the expertise emerges, at least in part, as the result of high motivation and years of practice. Perhaps the same is true for our special skills with the pragmatics of language.

A different way to address the origin of the mindreading abilities that underlie word learning is through the study of pathology. Suppose there is a single theory of mind capacity that underlies intentional inference in general, including the sorts of inference that go on in word learning. If so, then one should not find individuals who have intact word learning abilities but problems in non-linguistic theory of mind tasks, or vice-versa. On the other hand, if there really is a dedicated theory of mind mechanism for language, then one might expect to find such dissociations, people who are impaired in one domain but spared in another.

Such people might exist. One of the diagnostic features of Asperger's syndrome - a disorder sometimes conceptualized as a mild form of autism-is normal linguistic development. One might see this as showing that one can have a fully intact ability for intentional interpretation in the domain of language, while having an overall deficit in non-linguistic theory of mind. But there is another way to make sense of this population. There may be a continuum of theory of mind abilities, running from a severely autistic individual to a normal unimpaired person. And the extent of the language deficit is a direct function of the severity of the theory of mind deficit. A serious theory of mind deficit might leave children without the ability to orient preferentially to speech, share attention, or follow eye gaze, and they might never be able to grasp the notion of an arbitrary sign, leading to no word learning at all. A less severe impairment might make word learning possible, but limited and idiosyncratic. And in some cases, the theory of mind impairment might be sufficiently mild so as to leave word learning fairly unimpaired, although such individuals might still have problems with aspects of language such as irony and metaphor — as is the case for individuals with Asperger's syndrome. This is the approach taken by Frith and Happé (1994), who suggest that those individuals who have relatively preserved language skills are the same individuals who tend to perform well on tasks designed to tap their understanding of the thoughts of other people.

I will end with a cliché: More research needs to be done. But in my defense, I will also note that this should be very different research than has been done in the past. Through most of our intellectual history, word learning has been studied in the context of associative learning, and much contemporary research still adopts this perspective. But there is a different perspective one can take. Learning a word, after all, is a social act. When children learn that rabbits eat carrots they are learning something about the external world, but when they 
learn that rabbit refers to rabbits, they are learning an arbitrary convention shared by a community of speakers, something that is true only because of the contents of other people's minds. Even if we knew nothing else, this simple fact should make us sympathetic to the idea that theory of mind might be intimately related to the process of word learning, and should motivate further research along these lines.

\author{
Department of Psychology \\ Yale University
}

\title{
References
}

Alvarez, A. 2001: Poker: Bets, bluffs, and bad beats. San Francisco, CA: Chronicle.

Augustine, St. 398/1961: Confessions. New York: Penguin Books.

Baldwin, D.A. 1991: Infants' contribution to the achievement of joint reference. Child Development, 62, 875-890.

Baldwin, D.A. 1993: Infants' ability to consult the speaker for clues to word reference. Journal of Child Language, 20, 395-418.

Baldwin, D.A. 1995: Understanding relations between constraints and a socio-pragmatic account of meaning acquisition. Paper presented at the biennial meeting of the Society for Research in Child Development, Indianapolis, Indiana.

Baldwin, D.A., Markman, E.M., Bill, B., Desjardins, R.N., Irwin, J.M. and Tidball, G. 1996: Infants' reliance on a social criterion for establishing word-object relations. Child Development, 67, 3135-3153.

Baldwin, D.A. and Moses, L.M. 1994: Early understanding of referential intent and attentional focus: Evidence from language and emotion. In C. Lewis and P. Mitchell (eds.) Children's early understanding of mind: Origins and development. Hillsdale, NJ: Erlbaum.

Baron-Cohen, S., Baldwin, D.A. and Crowson, M. 1997: Do children with autism use the speaker's direction of gaze strategy to crack the code of language? Child Development, 68, 48-57.

Baron-Cohen, S., Leslie, A.M. and Frith, U. 1985: Does the autistic child have a 'theory of mind'? Cognition, 21, 37-46.

Birch, S. and Bloom, P. (in press). Preschoolers are sensitive to the speaker's knowledge when learning proper names. Child Development.

Bloom, L. 1994: Meaning and expression. In W. Overton and D. Palermo (eds.) The ontogenesis of meaning. Hillsdale, NJ: Erlbaum.

Bloom, P. 1994: Possible names: The role of syntax-semantics mappings in the acquisition of nominals. Lingua, 92, 297-329.

Bloom, P. 1996: Intention, history, and artifact concepts. Cognition, 60, 1-29.

Bloom, P. 2000: How children learn the meanings of words. Cambridge, MA: MIT Press.

Bloom, P. and Markson, L. 1998: Intention and analogy in children's naming of pictorial representations. Psychological Science, 9, 200-204.

(C) Blackwell Publishers Ltd. 2002 
Carey, S. and Bartlett, E. 1978: Acquiring a single new word. Papers and Reports on Child Language Development, 15, 17-29.

Clark, E.V. 1998: Conceptual perspective and lexical choice in acquisition. Cognition, 64, 1-37.

Diesendruck, G. and Markson, L. (2001). Children's Avoidance of Lexical Overlap: A Pragmatic Account. Developmental Psychology, 37, 630-641.

Frith, U. and Happé, F. 1994: Language and communication in autistic disorders. Philosophical Transactions of the Royal Society of London B, 346, 97-104.

Gelman, S. and Bloom, P. 2000: Young children are sensitive to how an object was created when deciding what to name it. Cognition, 76, 91-103.

Gentner, D. and Boroditsky, L. 2001: Individuation, relativity and early word learning. In M. Bowerman and S. Levinson (eds.) Conceptual development and Language acquisition. Cambridge: Cambridge University Press.

Gleitman, L.R. 1990: The structural sources of word meaning. Language Acquisition, $1,3-55$.

Goodman, N. 1983: Fact, fiction, and forecast. Cambridge, MA: Harvard University Press.

Grice, H.P. 1975: Logic and conversation. In P. Cole and J.L Morgan (eds.) Syntax and semantics 3: Speech Acts. New York: Academic Press.

Kanner, L. 1943: Autistic disturbances of affective contact. Nervous Child, 2, 217-250.

Kobayashi, H. 1998: How 2-year-old children learn novel part names of unfamiliar objects. Cognition, 68, B41-B51.

Lieven, E.V.M. 1994: Crosslinguistic and crosscultural aspects of language addressed to children. In C. Gallaway and B.J. Richards (eds.) Input and interaction in language acquisition. Cambridge: Cambridge University Press.

Locke, J. 1690/1964: An essay concerning human understanding. Cleveland: Meridian Books.

Macnamara, J. 1972: Cognitive basis of language learning in infants. Psychological Review, 79, 1-13.

Macnamara, J. 1982: Names for things: A study of human learning. Cambridge, MA: MIT Press.

Maratsos, M. 1991: How the acquisition of nouns may be different from that of verbs. In N. Krasnegor, D. Rumbaugh, R. Schiefelbusch and M. Studdert-Kennedy (eds.) Biological and behavioral determinants of language development. Hillsdale, NJ: Erlbaum.

Markman, E.M. 1989: Categorization and naming in children. Cambridge, MA: MIT Press.

Markman, E.M. 1992: Constraints on word learning: Speculations about their nature, origins, and domain specificity. In M.R. Gunnar and M.P. Maratsos (eds.) Modularity and constraints on language and cognition: The Minnesota Symposium on Child Psychology. Hillsdale, NJ: Erlbaum.

Markman, E.M. and Wachtel, G.F. 1988: Children's use of mutual exclusivity to constrain the meaning of words. Cognitive Psychology, 20, 121-157.

Markson, L. 1999: Fast mapping. Unpublished doctoral dissertation, University of Arizona. 
Markson, L. and Bloom, P. 1997: Evidence against a dedicated system for word learning in children. Nature, 385, 813-815.

Meltzoff. A.N. 1988: Infant imitation after a 1-week delay: Long term memory for novel acts and multiple stimuli. Developmental Psychology, 24, 470-476.

Meltzoff, A.N. 1995: Understanding the intentions of others: Re-enactment of intended acts by 18-month-old children. Developmental Psychology, 31, 838-850.

Merriman, W.E. and Bowman, L.L. 1989: The mutual exclusivity bias in children's word learning. Monographs of the Society for Research in Child Development, 54 (Serial No. 220).

Mervis, C.B., Golinkoff, R.M. and Bertrand, J. 1994: Two-year-olds readily learn multiple labels for the same basic-level kind. Child Development, 65, 1163-1177.

Plunkett, K. 1997: Theories of early language acquisition. Trends in Cognitive Sciences, 1, 146-153.

Quine, W.V.O. 1960: Word and object. Cambridge, MA: MIT Press.

Saussure, F. de 1916/1959: Course in general linguistics: New York: McGraw Hill.

Schieffelin, B.B. 1985: The acquisition of Kaluli. In D.I. Slobin (ed.) The crosslinguistic study of language acquisition Volume 1: The data Hillsdale, NJ: Erlbaum.

Spelke, E.S. 1994: Initial knowledge: Six suggestions. Cognition, 50, 443-447.

Sperber, D. and Wilson, D. 1986: Relevance: Communication and cognition. Cambridge, MA: MIT Press.

Sperber, D. 2000: Metarepresentations in an evolutionary perspective. In D. Sperber (ed.) Metarepresentations. New York: Oxford University Press.

Tomasello, M. and Akhtar, N. 1995: Two-year-olds use pragmatic cues to differentiate reference to objects and actions. Cognitive Development, 10, 201-224.

Woodward, A.L., Markman, E.M. and Fitzsimmons, C.M. 1994: Rapid word learning in 13- and 18-month-olds. Developmental Psychology, 30, 553-566. 